\title{
Individualized Agent Interactions
}

\author{
Ionut Damian, Birgit Endrass, Peter Huber, \\ Nikolaus Bee, and Elisabeth André \\ Human Centered Multimedia, Institute of Computer Science, \\ Augsburg University, 86159 Augsburg, Germany
}

\begin{abstract}
Individualized virtual agents can enhance the user's perception of a virtual scenario. However, most systems only provide customization for visual features of the characters. In this paper, we describe an approach to individualizing the non-verbal behavior of virtual agents. To this end, we present a software framework which is able to visualize individualized non-verbal behavior. For demonstration purposes, we designed four behavioral profiles that simulate prototypical behaviors for differences in personality and gender. These were then tested in an evaluation study.
\end{abstract}

\section{Introduction and Motivation}

Virtual simulations of social behavior can help visualize and analyze theoretical findings and assumptions in various sociological domains. However, software tools which provide such features often lack the flexibility and adaptability the researchers require. This shortcoming can also be observed in modern computer games where interactions between game characters follow a repetitive and predictable pattern which often leads to player immersion loss. For example, even though most MMORPGs (Massive Multiplayer Online Role Playing Games) allow the player to customize his or her character, this customization is only aesthetical, like clothing or hair style (Figure 1). The customization of behavioral characteristics, such as how the character gazes, how fast it gesticulates or what position and orientation it adopts during an interaction, is usually ignored.

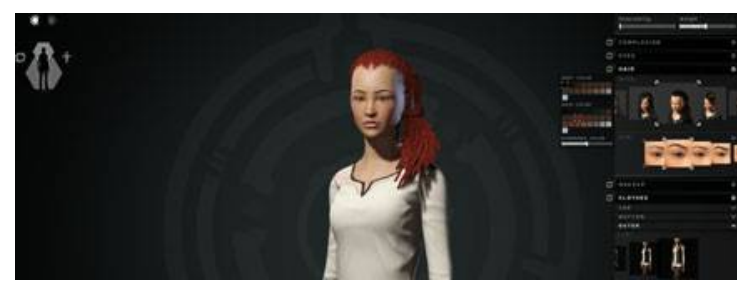

Fig. 1. The character customization tool in CCP's computer game "EVE Online"1

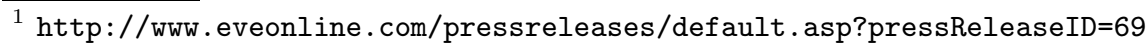


A solution to this problem is intelligent parametrization of the agents and the interactions. Using this approach, a designer can give each agent a set of specific parameters defining different aspects of its behavior, such as gazing techniques, positions during an interaction or gesture execution. These agents would then form realistic and diverse virtual environments.

The software framework Advanced Agent Animation (AAA) [1] is an implementation of the above mentioned approach. This framework is able to compute complex yet customizable interactions between virtual agents. This is done with the help of a powerful action parametrization system which covers all important aspects of an interaction: body location, body orientation, gestures, gazing and movement.

An evaluation study has also been conducted to offer some insight into the way people perceive differences in non-verbal behavior. The study investigated how well humans notice the individualized behavior of the virtual agents and whether they are able to recognize the different characteristics of non-verbal behavior the virtual agents simulate.

\section{Related Work}

There are a number of commercial software applications that can be used to simulate social behavior, however, most of them rely on explicit user input. For example, computer games, such as Second $\mathrm{Life}^{2}$, can be used to visualize agent interaction but the positioning and orientation of the agents requires continuous user input. Pedica argues in [24] that making users think about how to coordinate their virtual bodies every time they engage in a social interaction requires too much micromanagement. These actions should happen automatically without the user having to think about it. Automated generation of nonverbal behavior during agent interaction was first proposed in BodyChat [30]. Agents reacted to world events according to a preprogrammed rule set without the need for input from the user. The automated behavior consisted of conversational cues which could then be mapped to gaze actions and facial expressions. An improvement to BodyChat was the Spark system [29] with its incorporated BEAT engine [5]. It automated not only discourse related cues but also agent interaction related cues. Another system, Demeanour, presented by Gilles [8], was able to generate reactions in form of postures. Demeanour also provided means to customize the reaction generation by allowing the user to define avatar profiles which consisted of a set of parameters that described the avatar's attitude, friendliness or situational behavior. Both Spark and Demeanour lack an automated avatar positioning and orientation system, making either the user or the designer decide what position and orientation the avatars take during an interaction.

Systems like Spark or Demeanour do not actually deal with the existence of interactions between agents, they assume that these are happening and proceed

$\overline{2}$ http://www. secondlife.com 
with their computations based on this assumption. Pedica proposes, with the system CADIA Populus [24], a complex tool for simulating agent interactions. The virtual agents are able to move freely through a scene and interact with each other. The interactions in CADIA are defined as formations of virtual agents based on strong theoretical fundaments $[13,27,10]$. However, it lacks an animation system and a more realistic locomotion system, and the degree to which the generated behavior in CADIA can be customized is also unknown.

An approach to generating custom animations is presented by Neff and Kipp [21]. They describe a process in which gestures are first identified and annotated within a video corpus and are then passed to a gesture generation system. This process is able to generate unique gestures which can then be used to animate individualized virtual agents. However, the process is very complex and the annotation phase requires intensive user involvement and the availability of an appropriate video corpus. Pelachaud [25] presented a system for simulating behavior expressivity on the virtual agent Greta. The system uses a markup language, called APML (Affective Presentation Markup Language), to define the dialog and behavior the agents should execute. The APML also allows to control the expressivity [12] of the displayed animations.

A further notable example of animation customization is described by Chi et al. [6] in form of EMOTE. EMOTE is able to customize the execution of gestures by using the Laban [15] principles of Effort and Shape to alter the expressiveness of a gesture. This is done by varying the properties of the kinematic and spatial curve of specific keyframed actions. EMOTE can be described as a filter for animations as it can be applied to existing animations to alter their expressivity characteristics.

\section{Individualizing Non-verbal Behavior}

This paper focuses on the software framework for visualizing agent interactions Advanced Agent Animation (AAA) [1]. The framework automatically generates low-level behavior of virtual agents, such as moving their legs while walking or body positioning during an interaction, whereas the user or external systems are able to control the high-level behavior, such as which agent should interact with which agent or what gesture should an agent perform. The true power of the framework lies in its ability to customize most of the details of the simulation, from the stubbornness with which a virtual agent defends its personal space to the duration of the gazes the virtual agents throw each other. This is done with the help of action parametrization. Figure 2 shows a typical interaction in AAA featuring several virtual agents.

This section will describe the framework by presenting its four main features: formations, animations, gazing and movement. The focus will lie on the individualization parameters and how a designer can use them to create unique virtual agents that simulate specific characteristics of non-verbal behavior. 


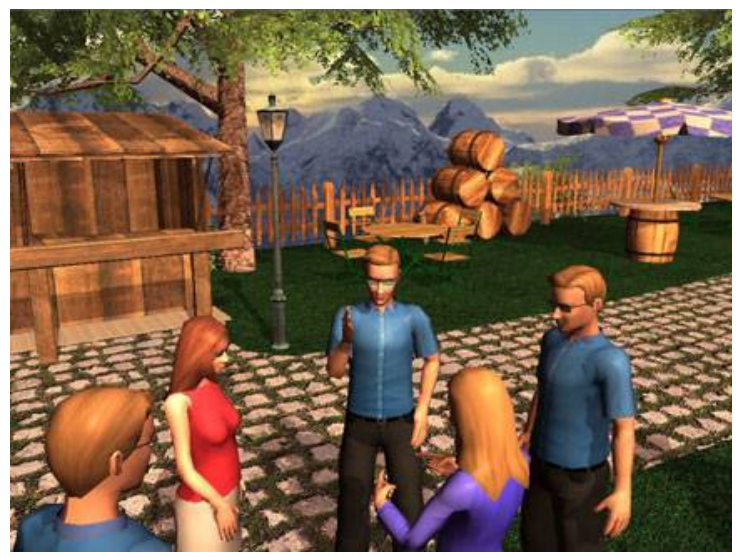

Fig. 2. The application Advanced Agent Animation (AAA) [1] showing an interaction between virtual agents

\subsection{Formation System}

The system uses F-formations [13] (Figure 3) to represent inter-agent interactions. Two or more agents can join together to form a formation. When joining a formation, a new agent automatically chooses a position inside the formation's p-space and orients itself so that its transactional segment intersects the transactional segments of the other members and the o-space of the formation. The transactional segment of an agent is the space in front of the agent where it conducts an activity, such as eating or gesticulating. Every member of a formation will try to satisfy its own preferences for interpersonal distance and orientation.

The positioning and orienting of an agent during an interaction happens automatically. This low-level behavior can be customized by altering an agent's parameters for interpersonal distances, willpower, deviation from the default orientation, and preferred formation type.

- Interpersonal distance: The distance between two agents is determined by the minIPDistance and maxIPDistance parameters of the two agents. Both agents will try to satisfy their two constraints on interpersonal distance during an interaction (Figure 4). For any two agents $\mathrm{A}$ and $\mathrm{B}$ which are part of the same formation, if A is closer to B then B's minIPDistance, B will move backwards until its minimal distance constraint is no longer violated. On the other hand, if $\mathrm{A}$ is further away from B then B's maxIPDistance, B will move towards A until the distance between them is smaller or equal its maximal distance constraint value.

- Willpower: If a conflict between two agents' preferences regarding interpersonal distance arises, the willpower parameter will define which agent will impose its constraints harder. The larger the willpower of an agent, the less inclined the agent will be to settle for a distance that does not satisfy its constraints. 


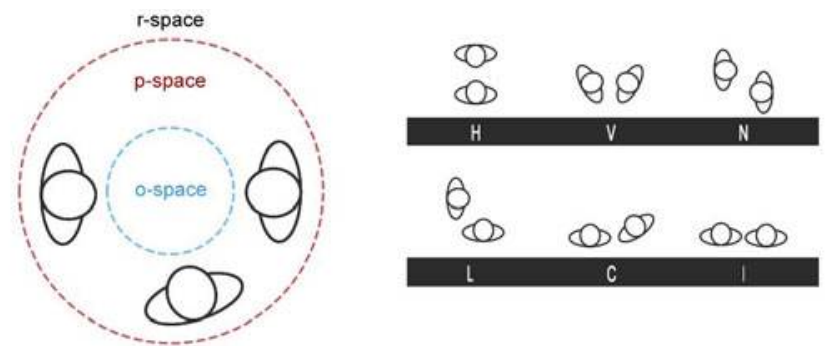

Fig. 3. The left structure represents Kendon's F-Formation [13] whereas the structures on the right represent the formation types for a pair of interlocutors described by Ciolek and Kendon [7]

- Deviation: This parameter will modify the body orientation of an agent during an interaction by deviating it from a normal state (Figure 4). The normal state of the body orientation is determined by the formation type. For instance, in an F-formation, the normal orientation of an agent is towards the center (o-space) of the formation. In this case, a 180 deviation will result in an agent that keeps its back to the formation center.
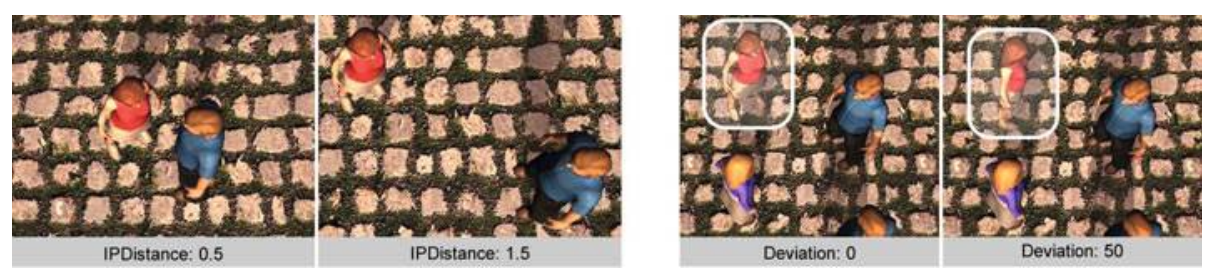

Fig. 4. The two screenshots on the left show the same agent formation but with varying interpersonal distance parameters whereas the screenshots on the right show varying deviation parameters

- Preferred formation: The preferred formation type controls which type of formation an agent will choose when engaging with another agent. The system uses Kendon's F-formation system [13] but can also reproduce the formation shapes described by Ciolek and Kendon [7]. Figure 3 shows illustrations for each formation type. For instance, if an agent $\mathrm{A}$ has a preferred formation type I, when an agent B engages in an interaction with A, they will go to an I-shaped (side-by-side) arrangement.

\subsection{Animation System}

The animation management system uses a powerful animation blending system which is able to realistically start the playback of an animation even if other animations are already being rendered on the agent. To achieve this, each 
animation is split into three phases: preparation, stroke and retraction [18]. The animation system also implements the action parametrization logic which enables the individualization of the expressivity [12] of every animation playback with the help of several parameters:

- Fluidity: Changing the length of the internal phases of an animation results in changes in the speed and softness of the transition from and to the animation. If an animation has a short preparation, then the blend-in phase will be shorter and sharper. The same principle applies for the retraction and the animation's blend-out phase. A short preparation and retraction also means that the stroke phase is longer and thus the phase that remains "untouched" is longer.

- Stroke repetitions: Each animation usually performs its stroke phase once. However, for animations such as beat gestures, it is very helpful to a designer to be able to specify how many times the stroke should be performed by the agent. This can be done with the help of the strokeRepetition parameter.

- Playback speed: Different individuals perform gestures with different speeds. This variation depends on many factors such as mood, age, gender, culture, and others. The systems allows the customization of the playback speed through the speed parameter. This controls the amount of animation frames the system performs for every application frame.
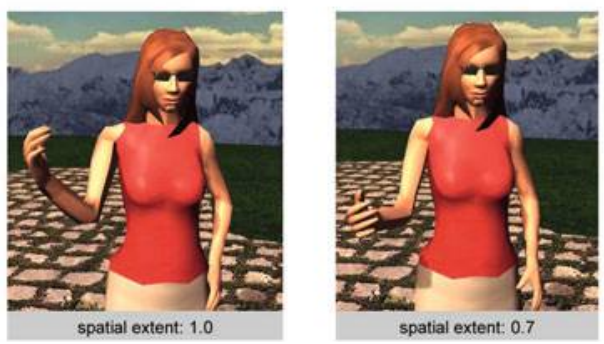

Fig. 5. Two screenshots showing the same animation playback, once with normal spatial extent and once with a lower spatial extent

- Spatial extent: The appearance of a gesture can vary with its spatial extent. High spatial extent means that the shape the gesture "draws" in the air will be large whereas a low spatial extent value correlates with small shapes. For instance, a "come here" gesture with a high spatial extent value would be performed with the hand at shoulder height and the arm far away from the body. However, the same gesture but with a lower spatial extent would result in the hand being lower and closer to the body (Figure 5).

\subsection{Movement System}

Agents are part of a virtual scene in which they are able to move freely. The agents can also orient themselves to face specific objects or coordinates. Each movement action can be customized with the help of the following parameters: 
- Walking speed: This parameter defines the speed with which an agent will traverse the distance between its current position and the target position.

- Movement animation: Each movement can have an animation which the system will perform while the movement is active. For instance, a walking animation should be performed during a slow locomotion whereas a fast locomotion would require a running animation.

\subsection{Gazing System}

The gazing system enables virtual agents to move their heads and eyes to gaze towards specific targets such as objects or other agents. Certain events automatically trigger gazing actions. For example, when a member of a formation speaks, the other members will gaze towards it. The user or external systems can also request gazing actions. All gazing actions can be customized with the help of the following parameters:

- Gazing speed: The gazing speed defines how fast an agent transitions from one gaze target to another. This parameter can be used to simulate certain emotions and moods of the agents, such as boredom or stress.

- Gaze duration: A gaze action is not a permanent state, humans use it most often as a reflex to observing something interesting and passes as soon as the individual looses interest in the target or another point of interest appears. The duration of gazing actions of a virtual agent can be customized with the gaze duration parameter. This parameter specifies how much time it has to pass until a gaze target expires. When this happens, the agent goes back to an older valid gaze target.

\subsection{Customization Examples}

The customization features presented in the previous sections can be used to simulate various agent characteristics. This section will present two dimensions of non-verbal behavior variations and how a designer can use the parameters of the framework to simulate them in virtual agents. For each dimension, we will also present a parameter profile represented by a table which provides a mapping of various theoretical findings to the customization parameters of the framework. The parameter profiles only focus on the parameters for which appropriate literature has been found.

3.5.1 Personality Differences. The personality of humans has a great impact on their behavior when interacting with other people. Based on the taxonomy of personality attributes presented by Norman [22], we will now try to present some theoretical findings regarding this observation. Argyle [3] and North [23] have postulated that the spatial behavior is different for introverts and extraverts with extraverts adopting closer distances during interactions. In addition to this, several studies show that extraversion correlates with higher spatial extent values when executing gestures $[14,16,26]$. The gesture execution 
Table 1. Example of parameter profiles for introversion and extraversion

\begin{tabular}{|l|l|l|l|}
\hline Parameter & Introversion & Extraversion & Reference \\
\hline minIPDistance & high & low & {$[3,23]$} \\
\hline maxIPDistance & high & low & {$[3,23]$} \\
\hline gesture speed & low & high & {$[16,4]$} \\
\hline gesture spatial extent & low & high & {$[14,16,26]$} \\
\hline gesture fluidity & low & high & {$[16,26,28]$} \\
\hline gaze duration & low & high & {$[19]$} \\
\hline
\end{tabular}

speed also tends to be higher for extraverts [16,4] making their gestures look more powerful. Studies have also shown that introversion negatively correlates with gestural smoothness or fluidity $[16,26,28]$. In the domain of oculesics, it has been found that extraverts use more eye contact [19] which correlates with higher values for the gaze duration parameter. Table 1 shows an example of how the parameters can be used to simulate introversion and extraversion.

Table 2. Example of parameter profiles for male and female agents

\begin{tabular}{|l|l|l|l|}
\hline Parameter & Female & Male & Reference \\
\hline minIPDistance & low & high & {$[11]$} \\
\hline maxIPDistance & low & high & {$[11]$} \\
\hline willpower & high & low & {$[2]$} \\
\hline deviation & low & normal & {$[9,11]$} \\
\hline preferred formation & F,H,V,N & all & {$[9,11]$} \\
\hline gesture spatial extent & low & high & {$[11]$} \\
\hline gaze duration & high & low & {$[9,11,17,20]$} \\
\hline
\end{tabular}

3.5.2 Gender Differences. A number of studies have shown that differences in non-verbal behavior exist between men and women. Hall [11] describes how women interact at closer distances than men. For us, this translates to smaller IPdistance values. Another interesting fact is that in mixed-sex interactions, the men also employ the close interpersonal distances that are typical for women [2]. We can simulate this with the willpower parameter. Other studies show that women are also more likely to adopt face to face orientations during an interaction $[9,11]$. The way humans execute gestures has also been found to vary between genders. Studies have shown that men tend to use larger spatial extent values when executing gestures [11]. Women also tend to gaze more during interactions $[9,11,17,20]$ which we can interpret as a larger value for the gaze duration parameter. Similar to the parameter profile presented in the previous section, Table 2 shows the parameter profile for the gender differences.

\section{Evaluation Study}

In order to evaluate the framework's customization features, we conducted a study in which we investigated how users perceive different parameter profiles. 
To this end, we implemented the previously described parameter profiles for the dimensions gender and personality into a series of scenarios which were then integrated into an evaluation study.

\subsection{Study Setup}

We analyzed the dimensions presented in the previous section: personality and gender. The personality dimension was featured with the values: introversion and extraversion; the gender dimension with female and male.

The study consisted of two videos for each dimension and one introduction video meant to familiarize the participant with the study. Every video showed an interaction between virtual agents. The two videos for a dimension showed identical interaction compositions and differed only through the usage of the customization parameters which were chosen based on the tables presented in Section 3. For each video, the participant had to grade the perceived behavior of the virtual agents relative to a dimension represented on a seven point scale, with the two dimension values on either side of the scale (Figure 6). Participants also had the possibility to leave a comment after each video. The study was conducted online and no restrictions for participation had been implemented.

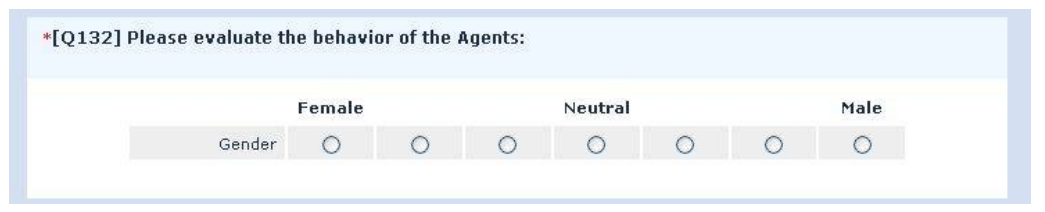

Fig. 6. A question from the online evaluation study

\subsection{Results}

For our analysis, we used two two sided t-tests, one for each dimension. A total of 133 participants ( 7 female and 126 male) took part in the study. They ranged in age from 14 to 49 , with an average of 22.03. As the study was held online and offered both in German and English, the participants also varied in nationality. A total of 29 nationalities have been recorded with the majority of the participants being of western culture.

Table 3 shows the mean and median value for each video and the p-value for each dimension. The tests yielded promising results. The $\mathrm{p}$ value $(p<0.001)$ of the personality test suggests that the participants generally recognized the simulated personality correctly. In case of the gender dimension, the results were also significant $(p=0.03)$. Furthermore, the average values, 4.03 for the video with simulated female behavior and 4.56 for the one with simulated male behavior, show that the video with simulated female behavior was in fact perceived as more female. However, a slight tendency towards the male-end of the scale can be observed. 
Table 3. Results of the survey showing the average and the p values of each dimension. The values ranged from 1 to 7 , where 1 represents most introverted or female, and 7 represents most extroverted or male.

\begin{tabular}{|r|l|l|l|l|}
\hline & \multicolumn{2}{|c|}{ Personality } & \multicolumn{2}{c|}{ Gender } \\
\hline & Introversion & Extraversion & Female & Male \\
\hline \hline mean & 2.41 & 5.96 & 4.03 & 4.56 \\
\hline median & 2 & 6 & 4 & 5 \\
\hline $\mathbf{p}$ & \multicolumn{2}{|c|}{$<0.001$} & \multicolumn{2}{c|}{0.03} \\
\hline
\end{tabular}

Overall, we observed that the perception was influenced by the appearance of the virtual agents. This effect was visible in the gender test, where, due to the male appearance of the virtual agents in both videos, the results show a tendency towards the male-end of the scale. Interestingly, the personality test did not show signs of such an influence. An explanation for this is that personality is appearance independent, as all types of people are affected by personality in the same way.

The evaluation showed that individualizing the non-verbal behavior of a virtual agent does in fact result in an altered perception of the simulated interaction and that users are likely to recognize simulated characteristics of non-verbal behavior in virtual agents.

\section{Conclusion}

The scope of this paper was to present an approach to individualizing interactions between virtual agents. To this end, we presented a software framework which is able to compute and visualize non-verbal behavior of virtual agents. The main strength of the framework is the flexibility of the behavior generation systems which allows a high level of behavior individualization. This allows designers to generate complex scenes populated by agents that behave in an unique way.

We presented four different usages of the individualization features which simulated two dimensions of non-verbal behavior variation: personality and gender. We then proceeded to test these in a perception study which yielded positive results regarding the ability of the participants to perceive the simulated characteristics of the non-verbal behavior.

A possible topic for future work would be to investigate how other dimensions can be simulated with the individualization parameters of the framework. It would also be interesting to analyze the degree to which each parameter of a profile influences the perception of the non-verbal behavior. We can conclude that individualizing the non-verbal behavior of virtual agents can be perceived by users and, if employed, can help designers better simulate agents and agent interactions. It is also plausible that such features would support user immersion in video games by giving users the opportunity to create avatars with which they can identify themselves more easily. However, our results have also shown that individualizing the non-verbal behavior should not replace the customization of the agents' appearance, but should be rather used to complement it. 
Acknowledgments. This work has been funded in part by the European Commission under the grant agreement DynaLearn (FP7-ICT-231526).

\section{References}

1. AAA: Advanced Agent Animation, http://hcm-lab.de/projects/aaa (last visited: 01.09.2011)

2. Andersen, P.R.: Nonverbal Communication: Forms and Functions. Mayfield (1998)

3. Argyle, M.: Bodily Communication, vol. 2. Methuen (1988)

4. Brebner, J.: Personality theory and movement. In: Individual Differences in Movement, pp. 27-41. MTP Press Limited (1985)

5. Cassell, J., Vilhjàlmsson, H.H., Bickmore, T.: BEAT: the behavior expression animation toolkit. In: SIGGRAPH 2001 Proceedings of the 28th Annual Conference on Computer Graphics and Interactive Techniques, pp. 477-486. ACM (2001)

6. Chi, D., Costa, M., Zhao, L., Badler, N.: The EMOTE model for effort and shape. In: Proc. Computer Graphics and Interactive Techniques, SIGGRAPH 2000, pp. 173-182. ACM Press/Addison-Wesley Publishing Co. (2000)

7. Ciolek, T.M., Kendon, A.: Environment and the spatial arrangement of conversational encounters. Sociological Inquiry 50(3-4), 237-271 (1980)

8. Gillies, M., Ballin, D.: Integrating autonomous behavior and user control for believable agents. Architecture, 336-343 (2004)

9. Guerrero, L.: Nonverbal involvement across interactions with same-sex friends, opposite-sex friends, and romantic partners: Consistency or change? Journal of Social and Personal Relationships 14, 31-59 (1997)

10. Hall, E.T.: The Hidden Dimension, vol. 6. Doubleday (1966)

11. Hall, J.A.: Nonverbal Sex Differences: Accuracy of Communication and Expressive Style. John Hopkins University Press (1984)

12. Hartmann, B., Mancini, M., Pelachaud, C.: Implementing expressive gesture synthesis for embodied conversational agents. Science 3881, 188-199 (2005)

13. Kendon, A.: Conducting Interaction: Patterns of behavior in focused encounters. Cambridge University Press (1990)

14. Knapp, M.L., Hall, J.A.: Nonverbal communication in human interaction. Holt, Rinehart and Winston New York (2009)

15. Laban, R., Lawrence, F.: Effort: Economy in Body Movement. Plays, Inc. (1974)

16. Lippa, R.: The nonverbal display and judgment of extraversion, masculinity, femininity, and gender diagnosticity: A lens model analysis. Journal of Research in Personality 32, 80-107 (1998)

17. McCormick, N., Jones, A.: Gender differences in nonverbal flirtation. Journal of Sex Education and Therapy 15, 271-282 (1989)

18. McNeill, D.: Hand and mind: What gestures reveal about thought. University of Chicago Press (1992)

19. Mehrabian, A.: Significance of posture and position in the communication of attitude and status relationships (1969)

20. Mulac, A., Studley, L.B., Wiemann, J.M., Bradac, J.J.: Male/female gaze in samesex and mixed-sex dyads gender-linked differences and mutual influence. Human Communication Research 13(3), 323-343 (1987)

21. Neff, M., Kipp, M., Albrecht, I., Seidel, H.-P.: Gesture modeling and animation based on a probabilistic re-creation of speaker style. ACM Transactions on Graphics 27(1), 1-24 (2008) 
22. Norman, W.T.: Toward an adequate taxonomy of personality attributes: Replicated factor structure in peer nomination personality ratings. Journal of Abnormal and Social Psychology 66(6), 574-583 (1963)

23. North, M.: Personality assessment through movement. Macdonald and Evans (1972)

24. Pedica, C., Vilhjálmsson, H.H.: Social Perception and Steering for Online Avatars. In: Prendinger, H., Lester, J.C., Ishizuka, M. (eds.) IVA 2008. LNCS (LNAI), vol. 5208, pp. 104-116. Springer, Heidelberg (2008)

25. Pelachaud, C.: Multimodal expressive embodied conversational agents, pp. 683689. ACM Press (2005)

26. Riggio, R.E., Friedman, H.S.: Impression formation: the role of expressive behavior. Journal of Personality and Social Psychology 50(2), 421-427 (1986)

27. Scheflen, A.E.: Human Territories: how we behave in space-time. Prentice-Hall (1976)

28. Takala, M.: Studies of psychomotor personality tests (1953)

29. Vilhjálmsson, H.H.: Animating Conversation in Online Games. In: Rauterberg, M. (ed.) ICEC 2004. LNCS, vol. 3166, pp. 139-150. Springer, Heidelberg (2004)

30. Vilhjàlmsson, H.H., Cassell, J.: BodyChat: Autonomous communicative behaviors in avatars. In: Proceedings of the 2nd International Conference on Autonomous Agents, pp. 269-276. ACM (1998) 